\title{
Modelos animais em psiquiatria: avanços e desafios
}

\author{
Emerson Arcoverde Nunes*1 \\ Jaime Eduardo Cecílio Hallak*2
}

Objetivos: Discutir os avanços e limitações do uso dos modelos animais nos transtornos psiquiátricos. Método: Uma revisão narrativa de artigos. Resultados: Diferentes modelos animais atualmente demonstram validade adequada para características especificas de determinados transtornos mentais. Conclusão: Resguardadas as devidas limitações que impossibilitam mimetizar sintomas psicopatológicos complexos em modelos animais, estes seguem como úteis ferramentas de estudo na psiquiatria.

Palavras-chave: Modelo animal, transtorno mental, psiquiatria, psicopatologia

${ }^{* 1}$ Faculdade de Medicina de Ribeirão Preto - Universidade de São Paulo - USP (São Paulo, SP, Br); Universidade Federal do Rio Grande do Norte - HUOL-UFRN (Natal, RN, Br).

*2 Faculdade de Medicina de Ribeirão Preto - Universidade de São Paulo - USP (São Paulo, SP, Br). 


\section{Introdução}

Há décadas os modelos animais têm sido usados regularmente em psiquiatria, tanto em pesquisas clínicas que tentam buscar um melhor entendimento da fisiopatologia dos transtornos mentais como em ensaios que buscam novas alternativas de tratamento psicofarmacológico (McGonigle, 2014). Mesmo com críticas da aplicabilidade de modelos animais para doenças em seres humanos (LaFollete and Sharks, 1993), é inegável a contribuição de tais modelos para uma melhor compreensão dos transtornos psiquiátricos (Insel, 2007). Com o passar dos anos, tais modelos foram sendo aperfeiçoados, buscando-se mimetizar quadros psicopatológicos complexos, tendo sido desenvolvidos modelos animais validados e amplamente utilizados para diferentes tipos de transtornos mentais, como depressão, ansiedade e modelos para esquizofrenia que, ao longo dos anos, ajudaram no melhor entendimento da fisiopatologia dos transtornos psiquiátricos e no desenvolvimento de novas opções terapêuticas psicofarmacológicas (Jones et al. 2011). Apesar disso, é evidente que desenvolver um modelo animal que mimetize por completo as características biopsicossociais de doenças humanas não é viável (McKinney, 2001). Assim, transpor alterações psicopatológicas de transtornos psiquiátricos em animais segue como um desafio, tendo em vista a complexidade de se reproduzir fenômenos complexos, como no caso de quadros psicopatológicos originalmente observados em seres humanos, para modelos utilizando animais evolutivamente inferiores, como no caso dos roedores e primatas, espécies frequentemente utilizadas em modelos animais nos transtornos psiquiátricos (Nestler \& Hyman, 2010). Lembremos ainda que uma das particularidades dos quadros psiquiátricos quando comparados a outros quadros na medicina clínica em geral, onde inclusive há mais tempo se 
utilizam modelos animais para estudo das mais diferentes doenças (Salgado \& Sandner, 2013), é que no caso dos transtornos psiquiátricos, mesmo com os avanços na busca por uma melhor compreensão da fisiopatologia de tais quadros, a falta de um substrato biológico objetivo que explique os quadros clínicos observados, juntamente com a subjetividade das alterações psicopatológicas presentes nos diferentes transtornos psiquiátricos (por exemplo, alucinações e delírios, sintomas que caracterizam experiências eminentemente presentes em seres humanos), adicionam dificuldades na busca de modelos que melhor caracterizem tais alterações em animais.

Desta forma, mesmo com modelos animais validados e amplamente utilizados na prática clínica nas últimas décadas, segue-se com a necessidade de constante reavaliação de tal validade dos modelos animais já desenvolvidos (McKinney, 2001), ressaltando-se inclusive a necessidade de busca por novos modelos que cada vez mais proporcionem uma melhor transposição de alterações psicopatológicas características de transtornos psiquiátricos para modelos em animais que possam ser reproduzidos em ambiente de pesquisa. Além disso, questiona-se até que ponto tais modelos animais seriam realmente capazes de representar modelos fidedignos e confiáveis de quadros tão complexos como os quadros psicopatológicos presentes nos transtornos psiquiátricos, com diferentes autores em publicações recentes tendo abordado de forma ampla o papel dos modelos animais nos transtornos psiquiátricos (Razafsha et al., 2013; McGonigle, 2014), favorecendo uma discussão mais profunda sobre o assunto.

Assim, o objetivo do presente trabalho é o de discutir até que ponto os modelos animais podem ser úteis para um melhor entendimento dos transtornos mentais em humanos, isto por meio de uma revisão de publicações que tratam do assunto, tentando-se abordar pontos que demonstrem o quanto se avançou até o momento no desenvolvimento dos modelos animais em psiquiatria, e possíveis lacunas ainda presentes neste campo do conhecimento.

\section{Metodologia}

Uma busca nas bases de dados eletrônicas Pubmed e Scielo foi feita, sem limite de tempo, utilizando-se as palavras-chave: "animal model", psychiatry, depression, mood disorder, schizophrenia and anxiety. Buscas adicionais nas referências de artigos selecionados foram feitas para ampliar o alcance das buscas feitas nas bases eletrônicas. Artigos encontrados que tratassem do tema foram revisados e utilizados na formulação de discussão sobre o papel dos modelos animais em diferentes transtornos psiquiátricos, a saber, esquizofrenia, transtornos de ansiedade e transtornos de humor. 


\section{OBSERVANDO A PSIQUIATRIA}

\section{Resultados e discussão}

\section{Validade dos modelos animais em psiquiatria}

Um aspecto importante para se avaliar a utilidade dos modelos animais é a validade. Dentre os tipos de critérios de validade, três foram propostos como de maior relevância para os modelos animais em transtornos psiquiátricos: validade aparente (também denominada validade de face), validade de construto e validade preditiva (Ellenbroek \& Cools, 1990). A validade aparente ou de face diz respeito a quão bem um modelo animal pode mimetizar os sintomas de uma determinada condição clínica, já a validade de construto está relacionada com a habilidade de um modelo reproduzir aspectos fisiopatológicos de determinada doença, ou seja, que remeta aos aspectos da biologia de determinada doença que se esteja tentando reproduzir. E, por último, a validade preditiva, que diz respeito à propriedade pela qual um modelo animal pode demonstrar resposta, em animais, aos agentes terapêuticos para determinada condição em humanos (McGonigle 2014). Apesar de se considerar que alguns modelos animais têm níveis razoáveis de validade, nota-se o desafio que pode ser utilizar tais modelos animais para mimetizar quadros complexos como os transtornos psiquiátricos. Uma característica única dos transtornos psiquiátricos que enfatiza tal complexidade é a natureza subjetiva da maioria dos sintomas presentes em transtornos mentais, que inclusive leva a uma dificuldade em se formular uma nosologia de psicopatologia uniforme (Kawa \& Giordano, 2012; Razafsha et al., 2013), o que influenciará de forma marcante no grau de validade que pode ser alcançado nos modelos animais em psiquiatria. Tendo em vista a ausência de marcador biológico ou alteração de imagem conclusiva para o diagnóstico dos transtornos psiquiátricos, avaliar a validade dos modelos animais em psiquiatria segue como um desafio para os pesquisadores.

Observa-se, no geral, que os modelos animais para transtornos psiquiátricos têm aceitáveis níveis de validade preditiva, ou seja, apresentam uma boa capacidade de demonstrar que terapêuticas aplicadas a animais podem se correlacionar com os efeitos observados em humanos. Por exemplo, no caso do teste do nado forçado (TNF), que ganhou aceitação baseado na sua capacidade para detectar a atividade de antidepressivos tricíclicos, mesmo que tenha necessitado de refinamento para detectar a atividade de inibidores seletivos da receptação de serotonina (Porsolt et al., 1977; Lucki, 1997; McGonigle, 2014). Já no que diz respeito à validade aparente (ou de face), notam-se níveis variáveis entre os diferentes modelos animais para transtornos psiquiátricos, dificilmente alcançando-se o mesmo nível observado no caso da validade preditiva. Como exemplo de modelo animal em psiquiatria com boa validade aparente, citamos o teste da inibição pré-pulso (prepulse inhibition 
- PPI), já tendo sido visto que pacientes com diagnóstico de esquizofrenia apresentam déficits no PPI que podem ser mimetizados pelo uso de anfetamina ou fenciclidina (PCP) em roedores (Powell et al., 2009). Apesar de exemplos positivos como este, um complicador para se alcançar níveis aceitáveis de validade aparente (ou de face) nos modelos animais para transtornos mentais, reside no fato da maioria dos sintomas psiquiátricos (delírios, alucinações, obsessões, culpa, entre outros) serem particularmente experiências humanas, podendo assim apenas serem inferidas em modelos animais, guardando-se significativas limitações. Sendo assim, não precisamos apontar para a dificuldade de relacionarmos um comportamento anormal em roedores como análogo a um comportamento anormal em humanos, de forma que atualmente existe um consenso de que o desenvolvimento de um modelo animal que reproduza uma síndrome psiquiátrica por completo, como no caso da esquizofrenia ou no caso dos transtornos de humor, é virtualmente impossível, reforçando o quão desafiador pode se tornar obter alto nível de validade aparente nos modelos animais em psiquiatria (Nestler \& Hyman, 2010; Salgado \& Sandner, 2013).

Já para a validade de construto, notamos uma dificuldade adicional, tendo em vista que mesmo utilizando-se exames complementares ou de imagem como auxiliares nos diagnósticos em psiquiatria, há falta de marcadores biológicos que possam objetivamente demonstrar o diagnóstico de transtornos psiquiátricos. Desta forma, temos de utilizar escalas e protocolos para diagnóstico das alterações mentais observadas, para daí formularmos um diagnóstico, o que pode ser ainda mais complexo de se fazer em modelos animais, dificultando por maneira a verificação de um nível adequado de validade de construto nestes modelos animais em psiquiatria (Razafsha et al., 2013). Apesar disso, recentemente, alterações moleculares e em circuitos biológicos têm sido identificadas tanto em modelos animais como concomitantemente em humanos com esquizofrenia e depressão (Kluge et al., 2011).

Desta forma, nota-se o desafio que pode ser para um modelo animal em psiquiatria ser considerado adequado na reprodução dos quadros presentes em transtornos psiquiátricos, pois para isto deverá apresentar similaridades de validade de construto, validade aparente e validade preditiva, para daí poder representar um robusto modelo animal para determinado transtorno psiquiátrico (Chadman et al., 2009).

\section{Endofenótipos e os modelos animais em psiquiatria}

Observando-se tal dificuldade de se reproduzir em modelos animais os fenômenos mentais presentes nos transtornos mentais, as pesquisas têm se concentrado em focar em traços discretos que possam ser estudados em animais de laboratório e ou ligados a alterações genéticas específicas. Assim, tem ganhado terreno na pesquisa em psiquiatria o termo endofenótipo, referente ao conceito de fatores 
fenotípicos hereditários que refletem componentes discretos dos processos fisiopatológicos, mais relacionados a genes predisponentes do que a síndrome psiquiátrica por completo (Gottesman \& Gould, 2003; Salgado \& Sandner, 2013). Uma das principais limitações do uso dos endofenótipos na caracterização de sintomas psiquiátricos em modelos animais é a falta de especificidade de tais endofenótipos para determinada síndrome psiquiátrica, podendo-se dar como exemplo o PPI, endofenótipo amplamente utilizado como modelo animal em esquizofrenia, que também está alterado na doença de Alzheimer, doença de Huntington e transtorno afetivo bipolar (Nestler \& Hyman, 2010). Além do seu uso nos modelos genéticos em psiquiatria, os endofenótipos são utilizados como variáveis dependentes em uma ampla gama de abordagens experimentais em psiquiatria, como em modelos farmacológicos, por lesão ou ambientais. Este tipo de abordagem leva em consideração que existe uma variável de controle baseada em uma hipótese etiopatogênica ou fisiopatológica específica para determinado transtorno mental, e o endofenótipo servindo como variável dependente. Como exemplo podemos citar modelos que utilizam privação social como variável para se avaliar seus efeitos sobre determinado endofenótipo de interesse (Salgado et al., 2006; Salgado \& Sandner, 2013). Mesmo assim, a desconstrução de transtornos mentais tão complexos e heterogêneos, tanto em etiologia como em sua fenomenologia, pode ser um complicador a mais para a validação dos modelos animais como ferramentas que adequadamente reproduzam os sintomas presentes em humanos portadores de transtornos mentais.

\section{Modelos animais comumente utilizados na pesquisa de transtornos mentais}

\section{Depressão}

A maioria dos modelos animais de depressão envolvem uma exposição aguda ou crônica a um estressor específico, a fim de provocar um ou mais sintomas do transtorno, sendo atualmente um dos mais populares e utilizados modelos de depressão o teste do nado forçado (TNF), inicialmente descrito por Porsolt e colaboradores, que se baseia na observação de que ratos colocados em um cilindro sem possibilidade de fuga, preenchido com água, adotam uma postura imóvel após tentativas iniciais de fuga (Porsolt et al., 1977; Cryan et al., 2002). Tal comportamento pode ser interpretado como uma forma de desamparo aprendido ou comportamento de desespero e o tempo gasto tentando escapar pode ser aumentado pelo uso de antidepressivos (McGonigle, 2014). Para demonstrar o potencial antidepressivo dos inibidores seletivos da receptação de serotonina, foi necessária uma adaptação do modelo para que se reconhecesse o tempo nadando e tentando escalar 
como comportamentos de fuga (Lucki, 1997). É um dos modelos mais difundidos e utilizados, tanto pela rapidez e fácil execução do teste como pela reprodutibilidade entre diferentes laboratórios. Apesar disso, existem limitações a este modelo, sendo uma das mais importantes a de que o estado de desamparo aprendido não é sustentado e, além disso, responde agudamente ao uso de antidepressivos, ao contrário do que se observa em pacientes deprimidos, que geralmente melhoram após algumas semanas do uso da medicação (Abelaira et al., 2013). Além disso, faz-se necessário um teste de campo aberto para avaliar a locomoção, para que se detecte falsos positivos nos casos de medicações que aumentem a locomoção, prolongando assim o tempo de tentativa de fuga, assim como os falsos negativos, no caso de medicações sedativas que promovam um menor tempo de tentativa de fuga nos animais. Teste semelhante que avalia os efeitos de medicações antidepressivas sobre o comportamento de desamparo aprendido é o teste de suspensão pela cauda, que também mede a duração do comportamento direcionado à fuga como uma variável associada a efeito antidepressivo (Cryan \& Mombereau, 2004). Ambos os testes são capazes de identificar o efeito terapêutico de todos os antidepressivos utilizados na prática clínica atualmente, lembrando que tanto os ansiolíticos como os antipsicóticos não são capazes de aumentar o tempo de comportamento relacionado à fuga em ambos os testes (Cryan \& Holmes, 2005).

Como citado anteriormente, uma das principais ressalvas a estes modelos é a falta de correlação do tempo de resposta observado nestes testes com o que se observa em pacientes com depressão, onde necessita-se de uso prolongado de antidepressivos para que se obtenha uma resposta terapêutica adequada. Ainda, notamos uma limitação no número de modelos animais de depressão que requeiram uso crônico de medicação antidepressiva para que se obtenha melhora, aproximando-se assim da resposta esperada nos pacientes com depressão. Os modelos mais utilizados que atendem a tal requisito são o modelo de bulbectomia olfatória e de estresse crônico moderado (chronic mild stress - CMS). Estes modelos envolvem procedimentos que resultam em mudanças duradouras no comportamento que podem ser revertidas pelo uso crônico de antidepressivos, mas não pelo uso agudo deles. No modelo da bulbectomia olfatória, a remoção cirúrgica dos bulbos olfatórios resulta no desenvolvimento de hiperatividade motora que pode ser revertida pelo uso crônico de antidepressivos, lembrando que neste modelo o uso agudo de tais medicamentos não demonstra ação terapêutica (Cryan \& Mombereau, 2004; Kelly et al., 1997; McGonigle, 2014). Apesar de tais características que aproximam este modelo ao que se observa na depressão maior em humanos, algumas características apresentadas pelos animais neste modelo representam diferenças significativas quando comparadas ao quadro clínico que este modelo se propõe a mimetizar. Podemos começar citando o fato da hiperatividade motora não ser um sintoma comum em casos de depressão, assim como o fato de, nos pacientes, geralmente 
necessitarmos de semanas para observar os efeitos terapêuticos das medicações; neste modelo necessitamos de apenas alguns dias, e ainda há a dificuldade de compreender a base neuroanatômica e fisiopatológica para a resposta das alterações comportamentais observadas e sua correlação com a depressão maior em humanos (McGonigle, 2014).

Já o modelo de estresse crônico moderado consiste na repetida e inesperada exposição a estressores moderados como alterações discretas na temperatura ambiente ou restrição de água, implementadas por um período de aproximadamente um mês (Cryan \& Mombereau, 2004; Willner, 1997; Katz, 1982; McGonigle, 2014). Assim, observa-se o aparecimento de diversas alterações comportamentais consideradas correlatos dos sintomas depressivos, como diminuição do comportamento de busca, alterações sexuais, agressividade, dentre outras, podendo todas estas serem revertidas pelo uso crônico de antidepressivos. Apesar de ser um modelo que melhor se assemelhe com o que se observa na depressão maior em humanos, tanto no que diz respeito às alterações apresentadas nos animais quanto ao que as provocou, o tempo necessário para se implementar tal modelo, o trabalho necessário para isto e o baixo nível de reprodutibilidade ao compararmos diferentes laboratórios, limita de alguma forma o uso deste modelo animal de depressão (McGonigle, 2014).

\section{Ansiedade}

No caso dos modelos animais de ansiedade, temos três categorias nas quais estes podem ser classificados, que são os testes etológicos (ou não condicionados), testes de conflito e testes cognitivos baseados ou de condicionamento clássico (Cryan \& Sweeney, 2011).

\section{Modelos de ansiedade por testes etológicos}

Estes modelos se baseiam em testes que combinam um comportamento não condicionado, naturalmente motivado, como a exploração do ambiente, com um estímulo aversivo para criar um conflito ou ansiedade no animal. Como no caso do amplamente utilizado teste do labirinto em cruz elevado (elevated plus maze EPM), talvez o modelo animal de ansiedade mais utilizado, inicialmente proposto por Handley e Mithani, e validado posteriormente por Pellow e File (Handley \& Mithani, 1984; Pellow \& File, 1986). Neste teste, o animal tem uma tendência natural a passar mais tempo nos dois braços fechados do que nos dois braços abertos sem paredes e elevados do labirinto. Os tratamentos com ansiolíticos aumentam o número de entradas e tempo que os animais permanecem nos braços 
abertos (Rodgers, 1997). Mesmo sendo um teste que controle alguns potenciais confundidores, como hiperlocomoção, este modelo é muito sensível a outras alterações como condições do ambiente (luz, barulhos e odores), assim como a diferentes raças de roedores (Griebel et al., 2000). Outros teste nesta mesma categoria incluem o labirinto em zero elevado, o teste da caixa claro-escuro, o teste em campo aberto, e o teste de enterrar defensivo, este último particularmente importante, pois é o único dentre os anteriormente citados capaz de detectar os efeitos ansiolíticos dos inibidores eletivos da receptação de serotonina - ISRS (Cryan \& Sweeney, 2011).

\section{Modelos de ansiedade por testes de conflito}

Os testes de conflito condicionado combinam um comportamento motivado, como beber ou comer, com um estímulo aversivo, como por exemplo um choque elétrico. O teste de conflito de Vogel, no qual ratos com privação de água recebem acesso ao consumo de água, mas o comportamento de beber a água é punido com um leve choque elétrico (Rodgers, 2010). Ansiolíticos (benzodiazepínicos por exemplo) produzem um aumento do consumo de água, a despeito da punição experimentada pelos animais. Como exemplo de outros testes incluídos nesta categoria podemos citar o teste de Geller-Seifter, o teste das quatro placas, dentre outros. O teste de Vogel e de Geller-Seifter são reconhecidos pelas suas baixas incidências de falsos positivos, mas são incapazes de detectar a ação ansiolítica de medicamentos ansiolíticos mais recentes como no caso dos inibidores seletivos da recaptação de serotonina (Cryan \& Sweeney, 2011), o que não é o caso do teste das quatro placas, este já com a capacidade de detectar os efeitos ansiolíticos dos ISRS.

\section{Testes cognitivos baseados ou de condicionamento clássico}

Tais testes utilizam condicionamento clássico para treinar o animal a associar um estímulo neutro, como um barulho, com um estímulo aversivo para alterar um comportamento natural (McGonigle, 2014). Como exemplo, podemos citar o teste do medo condicionado, onde o animal é treinado para associar um som ou local com um choque elétrico ou odor aversivo, com aumento nos níveis de comportamento de congelamento ou melhor resposta de sobressalto para o estímulo condicionado, funcionando como indicadores de um nível elevado de ansiedade (Borsini et al., 2002). Ansiolíticos podem reduzir os níveis de comportamento de congelamento ou resposta de sobressalto e o teste é sensível inclusive aos ISRS. Potenciais desvantagens de tais modelos residem no tempo necessário para o condicionamento dos 
animais e possíveis efeitos confundidores de medicações que possam interferir no limiar de dor, audição ou olfato, além de diferenças entre distintas raças de roedores (Balogh \& Wehner, 2003). Revisão recentemente publicada por Campos e colaboradores (2013) especificamente sobre os modelos animais de transtornos de ansiedade e estresse pode servir de fonte para maiores informações sobre o assunto.

\section{Esquizofrenia}

Existem três categorias diferentes de modelos animais de esquizofrenia, os farmacológicos, desenvolvimentais e genéticos, que levam em conta três diferentes grupos de sintomas: os sintomas positivos (alucinações e delírios), os sintomas negativos (anedonia, embotamento afetivo) e os sintomas cognitivos, como alterações na memória de trabalho e atenção (McGonigle, 2014). Os pacientes geralmente apresentam combinações heterogêneas de sintomas envolvendo estes três grupos de alterações, o que contribui para dificultar não só a formulação do diagnóstico como também o tratamento e o desenvolvimento de modelos animais.

\section{Modelos farmacológicos}

Os modelos farmacológicos estão entre os mais frequentemente utilizados, sendo os mais validados os que utilizam o tratamento agudo ou crônico com anfetamina ou fenciclidina (PCP), que promovem aumento na locomoção e déficits no PPI nos animais estudados (Mouri et al., 2007). Estes modelos foram desenvolvidos a partir das observações de que estas substâncias provocam alucinações e delírios quando utilizadas em humanos (Jones et al., 2011). Apesar de todos os medicamentos antipsicóticos atualmente utilizados serem ativos nestes modelos, existe um entendimento de que apenas os sintomas positivos são avaliados através destes modelos em questão, ficando os outros dois grupos de sintomas com limitada cobertura nestes testes farmacológicos (Jones et al., 2011).

\section{Modelos desenvolvimentais}

Como a esquizofrenia também é considerada um transtorno do neurodesenvolvimento, modelos que tentam reproduzir alterações desenvolvimentais por meio de exposições precoces a diferentes estressores, que causem alterações duradouras no comportamento dos animais que remetam aos sintomas da esquizofrenia, ganharam terreno ao longo dos anos. Podemos citar o modelo que utiliza o tratamento com 
metilazoximetanol em ratas grávidas, que ao serem expostas a este agente inibidor mitótico, geram descendentes com alterações comportamentais que se assemelham às presentes na esquizofrenia, como redução de estruturas neocorticais e límbicas, resposta motora aumentada ao uso de anfetamina, liberação aumentada de dopamina no núcleo accumbens e alterações no PPI (Jones et al, 2011; Moore et al., 2006). Existem outros dois modelos desenvolvimentais comumente utilizados, o de isolamento pós-desmame, o qual promove alterações cerebrais como redução do córtex pré-frontal além de diversas alterações comportamentais como hiperatividade, alterações no PPI e déficits cognitivos (Fone \& Porkess, 2008; Jones et al., 2011) e outro modelo que se baseia em lesão neonatal do hipocampo ventral, produzida após injeção local de substância tóxica no sétimo dia após o nascimento. Novamente, tais lesões produzem anormalidades que somente são notadas na puberdade dos animais (Jones et al., 2011; Lipska \& Weinberger, 1993), o que se correlaciona de maneira contundente ao observado em pacientes com esquizofrenia. Apesar disso, a validade preditiva de tais modelos desenvolvimentais ainda não foi adequadamente testada, além de que tais modelos são caros, demandando ainda um excesso de tempo e trabalho para serem implementados, o que pode limitar sua aplicabilidade. O modelo pela lesão por metilazoximetanol sofre de variações importantes dependentes do tempo no qual foi aplicada a intervenção, além de as alterações comportamentais serem sensíveis e poder serem revertidas apenas pelo manuseio dos animais. No geral, os modelos desenvolvimentais estão associados a maiores taxas de mortalidade, e mesmo que não sejam os melhores testes para novos antipsicóticos, podem ser úteis para a pesquisa dos mecanismos moleculares e fisiopatológicos na esquizofrenia (McGonigle, 2014).

\section{Modelos genéticos}

Mesmo com evidências que reforçam o caráter genético da esquizofrenia apontando para uma herança genética girando em torno de $80 \%$, não existe uma mutação gênica específica que esteja relacionada à miríade de sintomas que caracterizam um transtorno tão complexo como é a esquizofrenia (Aberg et al., 2013; Sun et al., 2010). Logo, uma série de diferentes genes candidatos têm sido associados a um risco aumentado para esquizofrenia. Tais genes, primordialmente envolvidos com plasticidade neuronal, função dopaminérgica e glutamatérgica e sinaptogênese, estão sendo alvos para produção de animais transgênicos que sirvam de modelo genético baseados na manipulação de diferentes genes. Podemos citar os modelos que utilizam alterações nos genes DISC-1 (do inglês, disrupted-in-schizophrenia 1), NRG1(neuregulina 1), e reelina, que estão envolvidos na sinaptogênese 


\section{OBSERVANDO A PSIQUIATRIA}

e plasticidade neuronal (Jones et al., 2011). Outros estudos envolvem diversos tipos de alterações gênicas, que produzem animais que apresentam alterações específicas que se assemelham às presentes na esquizofrenia, ainda que mais estudos sejam necessários antes de se estabelecer a validade e utilidade de tais modelos para a caracterização de sintomas negativos e cognitivos. Além disso, tais modelos genéticos produzem animais com fenótipos apresentando alterações aparentemente específicas demais para permitir caracterizar um transtorno tão complexo e heterogêneo como no caso da esquizofrenia (O'Tuathaigh et al., 2010).

\section{Conclusão}

Modelos animais para os transtornos mentais têm se mostrado úteis na pesquisa em psiquiatria, fornecendo dados importantes para o desenvolvimento de novas opções de tratamento e fornecendo pistas sobre mecanismos fisiopatológicos para os transtornos mentais. Apesar disso, a complexidade das alterações presentes nos transtornos psiquiátricos, a dificuldade de se transpor características de fenômenos puramente humanos, como as alucinações, delírios ou sentimentos de culpa para modelos animais evolutivamente inferiores, limita de maneira importante o alcance do que realmente é possível ser reproduzido nos modelos animais atualmente disponíveis.

\section{Referências}

Abelaira, H. M., Réus, G. Z., Quevedo, J. (2013). Animal models as tools to studey the pathophysiology of depression. Rev Bras Psiquiatr, Sipl. 2, S112-S120.

Aberg, K. A., Liu, Y., Buksza'r, J. et al. (2013). A comprehensive family-based replication study of schizophrenia genes. JAMA Psychiatry, 70(2),1-9.

Balogh, S. A. \& Wehner, J. M. (2003). Inbred mouse strain differences in the establishment of long-term fear memory. Behav Brain Res, 140(1-2), 97-106.

Borsini, F., Podhorna, J. \& Marazziti, D. (2002). Do animal models of anxiety predict anxiolytic-like effects of antidepressants? Psychopharmacol (Berl), 163, 121-141.

Campos, A. C., Fogaça, M. V., Aguiar, D. C. \& Guimarães, F. S. (2013). Animal models of anxiety disorders and stress. Rev Bras Psiquiatr, 35, S101-S111.

Chadman, K. K., Yang, M. \& Crawley, J. N. (2009). Criteria for validating mouse models of psychiatric diseases. Am J Med Genet B Neuropsychiatry Genet. 150B, 1-11.

Cryan, J. F. \& Holmes, A. (2005). The ascent of mouse: advances in modelling human depression and anxiety. Nat Rev Drug Discov, 4,775-790. 
Cryan, J. F., Markou, A. \& Lucki, I. (2002). Assessing antidepressant activity in rodents: recent developments and future needs. Trends Pharmacol Sci, 23(5), 238-245.

Cryan, J. F. \& Mombereau, C. (2004). In search of a depressed mouse: utility of models for studying depression-related behavior in genetically modified mice. Mol Psychiatry, 9, 326-357.

Cryan, J. F. \& Sweeney, F. F. (2011). The age of anxiety: role of animal models of anxiolytic action in drug discovery. Br J Pharmacol, 164, 1129-1161.

Ellenbroek, B. A. \& Cools, A. R. (1990). Animal models with construct validity for schizophrenia. Behav Pharmacol, 1, 469-490.

Fone, K. C. F. \& Porkess, M. V. (2008). Behavioural and neurochemical effects of post-weaning social isolation in rodents - relevance to developmental neuropsychiatric disorders. Neurosci Biobehav Rev, 32, 1087-1102.

Gottesman, I. I. \& Gould, T. D. (2003). The endophenotype concept in psychiatry: etymology and strategic intentions. Am J Psychiatry, 160, 636-645.

Griebel, G., Belzung, C., Perrault, G. \& Sanger, D. J. (2000). Differences in anxiety-related behaviours and in sensitivity to diazepam in inbred and outbred strains of mice. Psychopharmacol, 148, 164-170.

Handley, S. L. \& Mithani, S. (1984). Effect of alpha-adrenoceptor agonists and antagonists in a maze-exploration model of 'fear'-motivated behavior. Naunyn Schmiedebergs Arch Pharmacol, 327, 1-5.

Insel, T.R. (2007). From animal models to model animals. Biol Psychiatry, 62, 1337-1339.

Jones, C. A., Watson, D. J. \& Fone, K. C. (2011). Animal models of schizophrenia. Br J Pharmacol, 164(4), 1162-1194.

Katz, R. J. (1982). Animal model of depression: pharmacological sensitivity of a hedonic deficit. Pharmacol Biochem Behav, 16, 965-968.

Kawa, S., Giordano, J. (2012). A brief historicity of the Diagnostic and Statistical Manual of Mental Disorders: issues and implications for the future of psychiatric canon and practice. Philos Ethics Humanit Med, 7, 2.

Kelly, J. P., Wrynn, A. S. \& Leonard, B. E. (1997). The olfactrory bulbectomized rat as a model of depression: an update. Pharmacol Ther, 74, 299-316.

Kluge, W., Alsaif, M., Guest, P. C., Schwarz, E. \& Bahn, S. (2011). Translating potential biomarker candidates for schizophrenia and depression to animal models of psychiatric disorders. Expert Rev Mol Diagn, 11, 721-733.

La Follette, H., Shanks, N. (1993). Animal models in biomedical research: some epistemological worries. Public Aff, Q7, 113-130.

Lipska, B. K. \& Weinberger, D. R. (1993). Delayed-effects of neonatal hippocampal damage on haloperidol-induced catalepsy and apomorphine-induced stereotypic behaviours in the rat. Brain Res Dev Brain Res, 75, 213-222.

Lucki, I. (1997). The forced swimming test as a model for core and component behavioral effects of antidepressant drugs. Behav Pharmacol, 8, 523-532.

McGonigle, P. (2014). Animal models of CNS disorders. Biochem Pharmacol, 87, 140-149. 


\section{OBSERVANDO A PSIQUIATRIA}

McKinney, W. T. (2001). Overview of the past contributions of animal models and their changing place in psychiatry. Semin Clin Neuropsychiatry, 6, 68-78.

Moore, H., Jentsch, J. D., Ghajarnia, M., Geyer, M. A. \& Grace, A. A. (2006). A neurobehavioral systems analysis of adult rats exposed to methylazoxymethanol acetate on E17: implications for the neuropathology of schizophrenia. Biol Psychiatry, 60, 253-264.

Mouri, A., Noda, Y., Enomoto, T. \& Nabeshima, T. (2007). Phencyclidine animal models of schizophrenia: approaches from abnormality of glutamatergic neurotransmission and neurodevelopment. Neurochem Int, 51, 173-84.

Nestler, E. J. \& Hyman, S. E. (2010). Animal models of neuropsychiatric disorders. Nat Neurosci, 13, 1161-1169.

O’Tuathaigh, C., Kirby, B., Moran, P. \& Waddington, J. (2010). Mutant mouse models: genotype-phenotype relationships to negative symptoms in schizophrenia. Schizophr Bull, 36, 271-288.

Pellow, S. \& File, S. E. (1986). Anxiolytic and anxiogenic drug effects on exploratory activity in na elevated plus-maze: a novel test of anxiety in the rat. Pharmacol Biochem Behav, 24, 525-529.

Porsolt, R. D., LePichon, M. \& Jalfre, M. (1977). Depression: a new animal model sensitive to antidepressant treatment. Nature, 266, 730-732.

Powell, S. B., Zhou, X. \& Geyer, M. A. (2009). Prepulse inhibition and genetic mouse models of schizophrenia. Behav Brain Res, 204, 282-294.

Razafsha, M., Behforuzi, H., Harati, H., Wafai, R. Al., Khaku, A., Mondello, S., Goldb, M. S., Kobeissy, F. H. (2013). An updated overview of animal models in neuropsychiatry. Neurosci, 240, 104-218.

Rodgers, R. (2010). Animal tests for anxiety. In G. F. Koob, M. Le Moal \& R. F. Thompson, (ed.). Encyclopedia of Behavioral Neuroscience (pp. 90-100). Oxford: Academic Press.

Rodgers, R. J. (1997). Animal models of 'anxiety': where next. Behav Pharmacol, 8, 477-496.

Salgado, J. V., Hetem, L. A. \& Sandner, G. (2006). [Experimental models of schizophrenia - a review]. Rev Bras psiquiatr, 28, 135-141.

Salgado, J. V. \& Sandner, G. (2013). A critical overview of animal models of psychiatric disorders: challenges and perspectives. Rev Bras Psiquiatr, 35, S77-S81.

Sun, J., Jia, P., Fanous, A. H., van den Oord, E., Chen, X. et al. (2010). Schizophrenia gene networks and pathways and their applications for novel candidate gene selection. PLoS One, 5, e11351.

Willner, P. (1997). Validity, reliability and utility of the chronic mild stress model of depression: a 10-year review and evaluation. Psychopharmacol, 134, 319-329.

\section{Resumos}

(Animal models in psychiatry: Advances and challenges)

Objectives: To discuss the advances and limitations of animal models in psychiatric disorders. Method: A narrative review of articles. Results: Different animal models 
currently demonstrate adequate validity for specific characteristics of certain mental disorders. Conclusion: Recognizing limitations that stay away from any mimicking of complex psychopathological symptoms in animal models, such models nonetheless represent useful tools in the study of psychiatry.

Key words: Animal model, mental disorder, psychiatry, psychopathology

(Les modèles animaux en psychiatrie: progrès et défis)

Objectifs: Examiner les progrès et les limites des modèles animaux pour l'étude des troubles psychiatriques. Méthode: Révision narrative d'articles. Résultats: De différents modèles animaux possèdent actuellement une validité suffisante par rapport aux caractéristiques spécifiques de certains troubles mentaux. Conclusion: Malgré le fait des limites qui ne permettent pas de reproduire les symptômes psychopathologiques complexes dans les modèles animaux, ceux-ci restent néanmoins des outils utiles d'étude en psychiatrie.

Mots clés: Modèle animal, troubles mentaux, psychiatrie, psychopathologie

(Los modelos animales en psiquiatría: avances y desafios)

Objetivos: Discutir los avances y las limitaciones de los modelos animales en los trastornos psiquiátricos. Método: Una revisión narrativa de artículos. Resultados: Los diferentes modelos animales actualmente demuestran validez adecuada para las características específicas de determinados trastornos mentales. Conclusión: Guardadas las debidas limitaciones que imposibilitan mimetizar los síntomas psicopatológicos complejos en modelos animales, estes siguen siendo herramientas útiles en el estudio de la psiquiatría.

Palabras clave: Modelo animal, transtorno mental, la psiquiatria, psicopatología

(Tiermodelle in der Psychiatrie: Fortschritte und Herausforderungen)

Ziele: die Fortschritte und Grenzen von Tiermodellen bei psychiatrischen Erkrankungen zu diskutieren. Methode: Eine narrative Übersichtsartikel. Ergebnisse: Verschiedene Tiermodelle zeigen, derzeit ausreichende Gültigkeit für Besonderheiten bestimmter psychischer Störungen. Fazit: Geschützte die geltenden Einschränkungen, die Mimik komplexe psychopathologische Symptome im Tiermodell die folgenden als nützliche Werkzeuge in der Studie der Psychiatrie nicht aus.

Schlüsselwörter: Tiermodell, psychische Störung, Psychiatrie, Psychopathologie 


\section{OBSERVANDO A PSIQUIATRIA}

Citação/Citation: Nunes, E. A., Hallak, J. E. C. (2014, setembro). Modelos animais em psiquiatria: avanços e desafios. Revista Latinoamericana de Psicopatologia Fundamental, 17(3), 528-543.

Editor do artigo/Editor: Prof. Dr. Cláudio E. M. Banzato e Dra. Rafaela Zorzanelli

Recebido/Received: 26.6.2014/ 6.26.2014 Aceito/Accepted: 10.7.2014 / 7.10.2014

Copyright: (C) 2009 Associação Universitária de Pesquisa em Psicopatologia Fundamental/ University Association for Research in Fundamental Psychopathology. Este é um artigo de livre acesso, que permite uso irrestrito, distribuição e reprodução em qualquer meio, desde que o autor e a fonte sejam citados / This is an open-access article, which permits unrestricted use, distribution, and reproduction in any medium, provided the original author and source are credited.

Financiamento/Funding: Os autores declaram não ter sido financiados ou apoiados / The authors have no support or funding to report.

Conflito de interesses/Conflict of interest: Os autores declaram que não há conflito de interesses / The authors declare that has no conflict of interest.

\section{Emerson Arcoverde Nunes}

Departamento de Neurociências e Ciências do Comportamento - Faculdade de Medicina de Ribeirão Preto, Universidade de São Paulo - FMRP-USP (Ribeirão Preto, SP, Br); Hospital Universitário Onofre Lopes, Universidade Federal do Rio Grande do Norte - HUOL-UFRN (Natal, RN, Br).

Av. Bandeirantes, 3900 - Monte Alegre $\mathrm{S} / \mathrm{N}-3^{\circ}$ andar Depto. de Neurociências e Ciência do Comportamento 14048-900 Ribeirão Preto, SP, Br e-mail: emerson_arcoverde@yahoo.com.br

\section{Jaime Eduardo Cecílio Hallak}

Departamento de Neurociências e Ciências do Comportamento - Faculdade de Medicina de Ribeirão Preto, Universidade de São Paulo - FMRP-USP (Ribeirão Preto, SP, Br)

Av. Bandeirantes, 3900 - Monte Alegre $\mathrm{S} / \mathrm{N}-3^{\circ}$ andar Depto. de Neurociências e Ciência do Comportamento 14048-900 Ribeirão Preto, SP, Br e-mail: jhallak@fmrp.usp.br / jechallak@gmail.com 